\title{
Sensación térmica percibida en vivienda económica y auto- producida, en periodo cálido, para clima cálido húmedo
}

\author{
Perceived thermal sensation in low cost and self- \\ produced dwellings, in warm periods, in a warm humid \\ climate
}

\section{Carmen Garcia Gomez \\ Gonzalo Bojórquez Morales \\ Pavel Ruiz Torres}

Carmen Garcia Gomez Facultad de Arquitectura Universidad Autonoma de Yucatan Yucatan - México CEP 97000

Tel.: +(52) 99 9924-1300 Ext. 120 E-mail: ggomez.carmen@gmail.com

Gonzalo Bojórquez Morales Facultad de Arquitectura Universidad Autonoma de Yucatan Yucatan - México
E-mail: gonzalobojorquez@uabc.edu.mx

Pavel Ruiz Torres Facultad de Arquitectura Universidad Autonoma de Yucatan Yucatan - México E-mail: pavelvvg@msn.com

Recebido em 03/04/11 Aceito em 24/11/11

\section{Resumen}

$\mathbf{E}$ 1 estudio de la sensación térmica percibida en la vivienda, permite establecer lineamientos de diseño para la habitabilidad térmica, con base en la percepción de los sujetos. En trabajos anteriores, se plantea que la adaptación de las personas en climas cálidos, está definida por preferencias según el estrato socioeconómico y cultural, basado en su tipología de vivienda. Se presenta la comparación entre dos investigaciones sobre confort térmico desarrolladas en Mérida, Yucatán, México, con clima cálido húmedo. Los sujetos de estudio fueron habitantes de vivienda de tipo económico y autoproducida. Se utilizó el enfoque de adaptación, se trabajó con base en la norma ISO 10551 (1995) y se registraron temperatura de bulbo seco, temperatura de globo negro, humedad relativa y velocidad de viento. Se estimó la temperatura neutral con el método de medias por intervalo de sensación térmica, y se compararon preferencia, aceptación y tolerancia del ambiente térmico. Los resultados de ambas investigaciones muestran variaciones en la temperatura neutral y rangos de confort térmico, además se presentan diferencias significativas en el nivel de adaptación, experiencia y expectativa, aún cuando las condiciones del ambiente térmico presentan similitudes.

Palavras-chave: Sensación térmica percibida. Adaptación psicológica. Aclimatación. Vivienda económica. Vivienda auto-producida. Clima cálido húmedo.

\section{Abstract}

The study of perceived thermal sensation in housing allows designers to set design guidelines for thermal occupancy based on the perception of the dwellers. Previous research work suggests that the adaptation of people in hot climates is determined by preferences according to their socioeconomic and cultural class, based on their type of housing. A comparison is presented between two investigations into thermal comfort carried out in Mérida, Yucatán, México, in a warm humid climate. The dwellers were residents of self-produced economic housing. The adaptive approach was used, with the ISO 10551 (1995) standard as a basis. Dry bulb temperature, black globe temperature, relative humidity and wind humidity were monitored. The neutral temperature was estimated using the Intervals Means of Thermal Sensation Method, and preference, acceptance and tolerance of thermal environment were compared. The results of both investigations show variations in the neutral temperature and thermal comfort ranges, as well as significant differences in the level of adaptation, experience and expectation, even when the thermal environment conditions present similarities.

Keywords: Perceived thermal sensation. Psychological adaptation. Acclimatization. Lowcost housing. Self-produced housing. Warm humid climate. 


\section{Introducción}

La adaptación térmica se genera en función de la interrelación de los parámetros físicos, personales, aspectos sociales y culturales, ya que estos establecen la relación entre el usuario y su ambiente térmico. Los factores del contexto y la experiencia térmica modifican la expectativa y preferencia de los usuarios de edificios (SINGH; MAHAPATRA; ATREYA, 2011)

El confort térmico estudiado con el enfoque de adaptación, sirve como referencia para establecer la expectativa de los usuarios y sus condiciones térmicas preferidas, que pueden implicar aumento en el costo de operación por climatización artificial de los edificios. Por lo anterior, la aplicación de normas como ASHRAE 55 (ASHRAE, 2004), es un factor importante en la decisión de la sostenibilidad de la construcción.

La aplicación de estrategias de adecuación climática en la edificación, con justificación en la toma de decisiones durante el proceso de diseño, hace necesario ampliar el conocimiento sobre adaptación térmica, por lo que se deben realizar trabajos de investigación que documenten el valor de las conductas voluntarias individuales e incluyan los aspectos sociales, económicos y culturales; que combinados permitan inferir la percepción personal del ambiente térmico.

El objetivo de este trabajo es presentar un estudio comparativo sobre confort térmico y percepción térmica, en dos tipos de vivienda con variación de factores sociales, económicos y culturales de la ciudad de Mérida, Yucatán, México, que presenta un clima cálido húmedo.

Según ASHRAE 55 (ASHRAE, 2004), el confort térmico es una respuesta subjetiva y se define como "[...] la condición mental en la cual se expresa satisfacción con el ambiente térmico [...]". Con respecto a lo anterior, Singh, Mahapatra y Atreya, (2011) establecen que un mismo ambiente térmico puede ser percibido de manera diferente, por distintas personas y que la condición de confort térmico puede variar de un ambiente térmico a otro según la experiencia y expectativa térmica de los usuarios.

El enfoque de adaptación considera en sus análisis distintos factores que influyen de manera particular en cada sujeto y en su percepción del ambiente térmico, por lo que los resultados no son valores puntuales, sino que se establecen rangos de confort térmico. Kwok y Rajkovich (2010) clasifican los factores en tres categorías:

(a) adaptación del comportamiento: se refiere al tipo de ropa y aumento o reducción del nivel de aislamiento por la misma, el movimiento del cuerpo o los objetos en sus alrededores inmediatos para crear un ambiente térmico más satisfactorio. Como adición o eliminación de ropa, cambio de postura, apertura o cierre de ventanas, cambio de temperatura de control en termostatos, uso de ventiladores, bloquear o redirigir el viento de los difusores y uso de persianas para bloquear la radiación solar no deseada;

(b) adaptación fisiológica: es la adaptación del organismo a los ambientes térmicos estresantes (caliente o frío). Se presenta como cambios fisiológicos en el metabolismo con diferentes respuestas de termorregulación como vasodilatación, vasoconstricción, escalofríos y sudoración. En general esto representa el fenómeno de aclimatación; y

(c) adaptación psicológica: se refiere a la percepción térmica que puede ser directa y significativamente modificada por la experiencia térmica y la expectativa de las condiciones en espacios con sistemas electromecánicos de acondicionamiento ambiental.

En términos prácticos, la adaptación se define como la capacidad de un ocupante en un edificio para realizar ajustes en el entorno local (adaptación de la conducta) o el estatus propio (psicológica y/o adaptación fisiológica). Entre las condiciones de confort térmico ideales establecidas en modelos estáticos y con condiciones térmicas ambientales que producen respuestas involuntarias, hay una oportunidad significativa para la adaptación (KWOK; RAJKOVICH, 2010).

\section{Tipos de vivienda}

El requerimiento de vivienda en México, es cubierto por dos tipos de acciones:

(a) vivienda formal: en la cual se realiza un trámite para que por medio de financiamiento se puede comprar una vivienda construida en serie; $y$

(b) vivienda informal: donde los propietarios adquieren un terreno y contratan mano de obra o ellos mismos construyen conforme a su capacidad económica.

La vivienda formal incluye a sectores de nivel económico que hacen vivienda por encargo, al sector medio que tiene financiamiento bancario y al nivel social que se hace a través de organismos estatales cuya función es ser facilitadores del otorgamiento de crédito, pues el sector privado actúa como actor principal de la producción en serie (PUEBLA, 2002). 
En el año 2000 el gobierno intentó disminuir el rezago habitacional, que ascendía a $382 \mathrm{mil}$ unidades anuales (GARCÍA-PERALTA, 2010), y acelerar la economía mediante la industria de la construcción para captar al sector asalariado de menores ingresos.

Esto se logró al instituir como política oficial la construcción en serie en la categoría denominada "vivienda económica" que considera un costo de hasta 117,063 veces el salario mínimo mensual (SMM) del Distrito Federal. En costos reales en Mérida, Yucatán, ese monto sirve para una construcción de aproximadamente $40 \mathrm{~m}^{2}$.

La vivienda económica, que es vivienda formal, está dirigida a trabajadores con ingreso diario individual o familiar de 1 a 3,9 veces SMM, que en Mérida representa $\$ 56,70$ pesos mexicanos (aproximadamente \$4,64 dólares USA). El terreno es de $8 \times 20$ metros, la construcción se hace con materiales durables, mano de obra especializada y se incluyen servicios como agua, electricidad, sistema de saneamiento de aguas negras, banquetas y pavimentación (ver Figura 1).

La vivienda auto-producida, que es vivienda informal, tiene un proceso de edificación individualizada, crecimiento por etapas a largo plazo, se construye al inicio con materiales perecederos, reciclados y de desecho y cambia a materiales más sólidos conforme evoluciona. Esto hace reflexionar en la capacidad de gestión y decisión, energía, creatividad y audacia pues los propietarios se encargan casi totalmente de la "producción", es decir consigue un terreno; se busca el financiamiento; diseñan o concibe la vivienda; compran los materiales y consiguen mano de obra para algunas etapas de la edificación (GARCÍA-GÓMEZ, 2006).

El terreno promedio es de 10x25 metros, en sus primeras etapas tienen un espacio para dormir y otro para comer y estar; la cocina y el baño se encuentran en el exterior separados de la zona habitable (ver Figura 2), sus dueños son predominantemente subempleados dedicados al comercio ambulante, personas que ejercen un oficio en sus viviendas y mujeres que realizan trabajo doméstico pagado, con un ingreso promedio de dos veces el salario mínimo diario $(\$ 113,40$ pesos mexicanos, aproximadamente \$9,28 dólares USA) (GARCÍA-GÓMEZ, 2000).
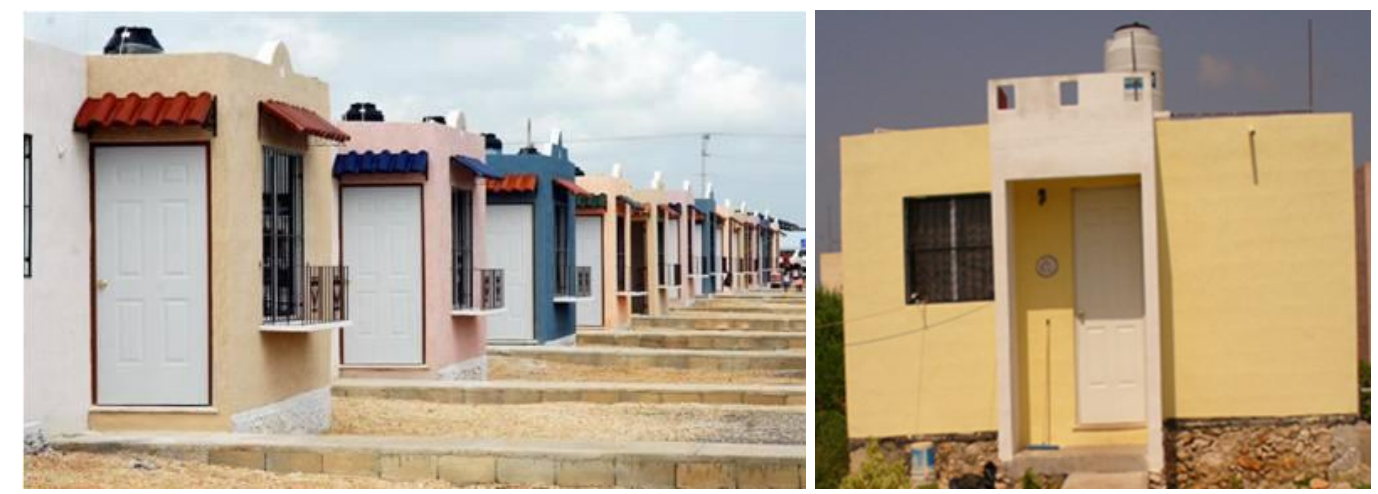

Figura 1 - Fotos de la vivienda económica en Mérida, Yucatán, México
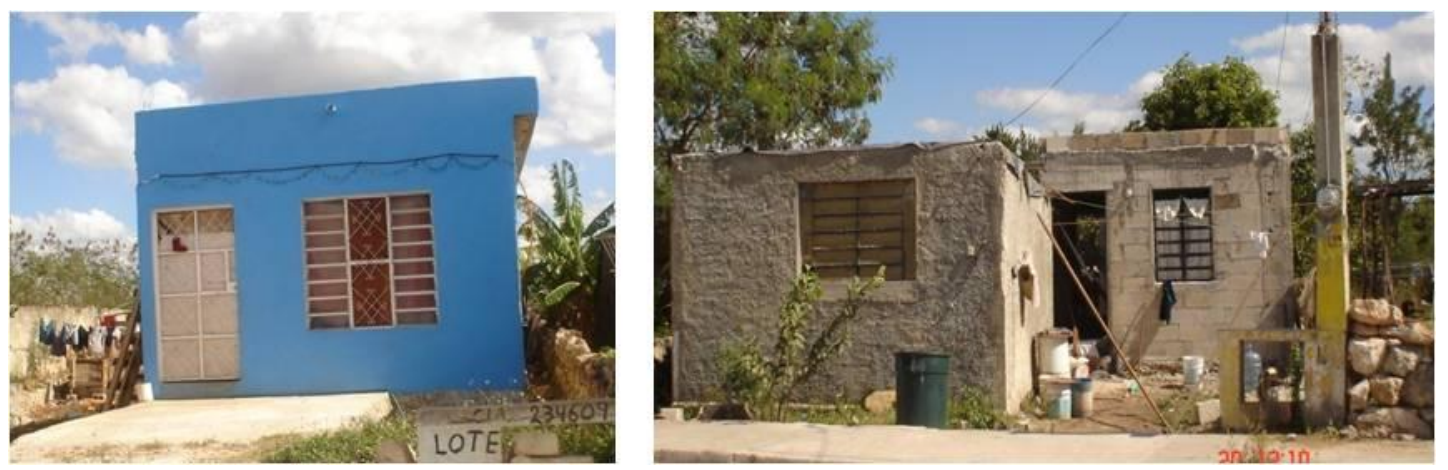

Figura 2 - Vivienda auto-producida en Mérida, Yucatán, México 
La autoproducción de vivienda genera a mediano plazo un espacio más adecuado a la realidad económica y social de los auto-productores, ya que la construcción generalmente es más grande que la vivienda económica, retoma el significado, la manera de vivir y la visión cultural de la realidad de sus habitantes, ya que sigue un patrón de producción social del espacio y además relaciona la casa con el terreno y responde a la adecuación con el medio ambiente a través del uso de vegetación como sombra, la construcción de la casa es en la parte central del terreno para favorecer la ventilación natural, y usan piedras apiladas de baja altura como división entre predios que permite el flujo de viento.

\section{Método}

El desarrollo de la investigación se presenta en cuatro apartados:

(a) diseño de la investigación;

(b) casos específicos de vivienda;

(c) variables ambientales; $y$

(d) estudio correlacional.

\section{Diseño de investigación}

El estudio pretende evaluar la capacidad de respuesta de sujetos de diferente estrato económico y sociocultural, bajo diferentes condiciones de ambiente térmico, en este caso los dos tipos de vivienda.

El enfoque de estudio fue determinado en función del objetivo y condicionantes de la investigación, según los parámetros de: origen de los datos, tipo de hábitat, reacciones a analizar, tipo de receptor de la información y nivel de análisis, todos los requerimientos coinciden con el enfoque de adaptación; el cual establece como parte de su aplicación el diseño de una investigación basada en correlaciones, lo que conlleva a un diseño de la investigación de tipo "no experimental".

La dimensión temporal fue de tipo transversal, ya que el análisis se desarrolló para el periodo cálido. El fenómeno estudiado fue la sensación térmica percibida por el usuario de espacios interiores de viviendas de tipo económico y auto-producida en clima cálido húmedo.

El estudio se realizó en Mérida, Yucatán, ciudad del sureste de México que se encuentra a una latitud de $20^{\circ} 59^{\prime} 00^{\prime \prime} \mathrm{N}$ y longitud $89^{\circ} 38^{\prime} 00^{\prime \prime} \mathrm{O}$, con una altura sobre el nivel del mar de nueve metros y temperaturas promedio de $26^{\circ} \mathrm{C}$, máximas de 39 ${ }^{\circ} \mathrm{C}$ y mínimas de $17{ }^{\circ} \mathrm{C}$ (GARCÍA-GÓMEZ, 2009).

\section{Casos específicos de vivienda}

Los objetos de estudio de este trabajo son la:

(a) vivienda económica; y

(b) vivienda auto-producida.

En el 2010, en Mérida se construyeron 10,416 viviendas de tipo económica y en lo que va del año 2011 se han construido 9,465 acciones por lo que se estima cifra del 2010 será superada, mientras que la vivienda de auto-producida cuenta con una existencia actual de 30,740 viviendas, según el Programa de Desarrollo Urbano de Mérida (AYUNTAMIENTO..., 2011).

\section{Variables ambientales}

La selección de variables ambientales a medir se basó en el efecto de las mismas en la sensación térmica percibida, así como en el análisis de casos de estudio sobre confort térmico en interiores. Para elegir los instrumentos de trabajo, se consideraron precisión y rangos de los mismos, la disponibilidad y accesibilidad, además de la complejidad de operación.

En el caso de las variables ambientales se revisaron trabajos de investigación realizados con el enfoque de adaptación, como Singh, Mahapatra y Atreya (2011), que realizaron un estudio de percepción del ambiente térmico en tres diferentes zonas climáticas del nordeste de la India en cuatro diferentes estaciones del año. Cao (2010), reportó un estudio durante el verano y el invierno en Beijing; bajo el concepto de la capacidad de adaptación de las personas ante el ambiente térmico, en espacios interiores de aulas y oficinas. Además se revisaron las normas ISO 7730 (ISO, 2005), ISO 10551 (ISO, 1995) y ASHRAE 55 (ASHRAE, 2004).

En lo que respecta a la precisión y rango, en temperaturas era necesario tener instrumentos con errores menores a $1{ }^{\circ} \mathrm{C}$, por el efecto que esta variación puede ocasionar en la sensación térmica percibida del usuario. Por otro lado, según García (1981) en Mérida, Yucatán se tienen registros de temperatura máxima extremas de $43{ }^{\circ} \mathrm{C}$ y un record histórico de $46{ }^{\circ} \mathrm{C}$, así como una mínima extrema de $4{ }^{\circ} \mathrm{C}$, por lo que se estableció un rango en los sensores de temperatura de 1 a $50{ }^{\circ} \mathrm{C}$. En el caso de la humedad relativa, en Mérida, existen valores promedios de máximas entre 40 y $90 \%$ con extremos hasta del 93\%. Por lo que consideraron rangos de medición entre el 35 y $100 \%$.

Se consideró que los instrumentos a utilizar fueran sencillos, sin requerir de una formación o capacitación de uso especializada, debido a que el estudio se basa en la aplicación de encuestas, las

102 Garcia Gomez, C.; Bojórquez Morales, G.; Ruiz Torres, P. 
cuales fueron aplicadas por estudiantes de la Facultad de Arquitectura de la Universidad Autónoma de Yucatán. Una de las principales características del equipo a utilizar fue que tuviera despliegue en pantalla de la información registrada para así poder anotar en las encuestas las condiciones ambientales en las que se desarrolló su aplicación.

Para el monitoreo en interiores se utilizó el equipo de monitor de estrés térmico QUESTemp 36, el cual se puede utilizar en ambientes en condiciones de trabajo o descanso, y uso general de la evaluación del ambiente térmico. Este equipo registra temperatura de bulbo seco, temperatura de bulbo húmedo, temperatura de globo negro, humedad relativa y velocidad de viento. Esta última se midió con un anemómetro omnidireccional para velocidades entre $\begin{array}{llll}0 & \text { y } & 20\end{array}$ metros por segundo, en incrementos de $0.1 \mathrm{~m} / \mathrm{s}$.

\section{Estudio correlacional}

Se desarrolló un estudio de tipo correlacional, con el enfoque de adaptación, en el período cálido, durante mayo a julio de 2007. Se utilizó un cuestionario basado en ISO 10551 (ISSO, 1995) y se midieron temperatura de bulbo seco, temperatura de globo negro, humedad relativa y velocidad de viento. El análisis de datos se llevó a cabo con el método de medias por intervalo de sensación térmica, propuesto por Gómez et al. (2007).

Los sujetos de estudio fueron hombres y mujeres entre 12 y 65 años de edad, no se incluyeron individuos con condiciones biológicas irregulares como enfermedades crónicas, embarazo, periodo de lactancia o periodo menstrual. Las encuestas fueron aplicadas en posición de pie o sentado sobre una silla o un banco ligero con el fin de reducir la afectación por aislamiento de mueble.

La aplicación de las encuestas fue en el periodo cálido, en un horario de 8:00 a 19:00 horas. Los instrumentos se colocaron al centro del espacio para uniformizar el efecto de radiación infrarroja de muros y cubiertas. Para sujetos de pie los sensores se colocaron a $1,20 \mathrm{~m}$ y para sujetos sentados $0,80 \mathrm{~m}$.

Se elaboró un cuestionario preliminar, se desarrolló un manual de aplicación de encuesta y del uso de instrumentos. Se hizo una prueba piloto y con base en los resultados obtenidos se procedió a las correcciones necesarias.

Los niveles de actividad de los habitantes de las viviendas fueron de tipo: pasivo, moderado e intenso, según la clasificación del trabajo de Fanger (1986); la tasa metabólica se estimó con base en la similitud de actividades desarrolladas (ver televisión, barrer, limpiar, etc.), con respecto al listado que presenta ISO 8996 ( ISO, 2005) en su tabla de actividades específicas.

El nivel de aislamiento por ropa, se estimó con base en una clasificación del tipo de vestimenta (cinco escalas, desde muy ligero a muy arropado), y se determinó el nivel de arropamiento promedio según el sexo del sujeto y escala de vestimenta, conforme a los valores que presenta ISO 9920 (ISO, 2007).

Se estimó de muestra, a partir del número promedio de personas por familia en Mérida y de la cantidad de viviendas ocupadas existentes en los sitios de estudio. Se calculó con una confiabilidad del $95 \%$ y precisión de los estimadores del $5 \%$, con la población blanco seleccionada se obtuvo una muestra de 150 encuestas por tipo de vivienda.

El proceso de selección de los sujetos fue de tipo determinístico. Se encuestó a todos los habitantes que cumplían con los parámetros siguientes:

(a) haber habitado en la casa cuando menos con un año;

(b) estar en el rango de edad; $y$

(c) no estar en las condiciones de exclusión antes mencionadas.

Algunas imágenes de aplicación de encuestas y equipo se presentan en la Figura 3.
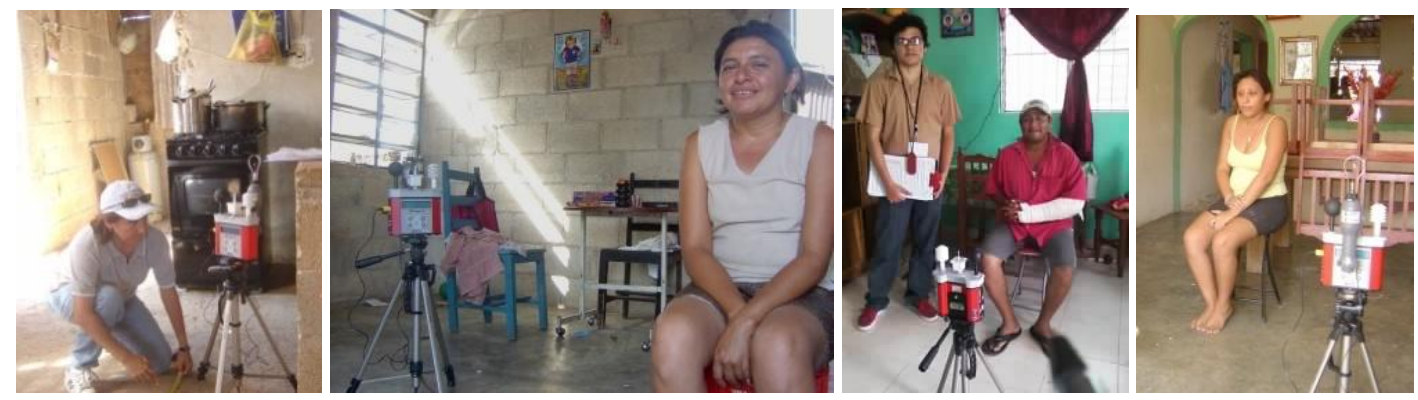

Figura 3 - Fotos de la aplicación de encuestas y monitor de estrés térmico 
Los fraccionamientos de vivienda económica considerados en el trabajo de campo fueron: Tixcacal Opichen y San José Tecoh. Aun cuando se cumplió con el horario estipulado en la metodología; es importante mencionar que el mayor porcentaje $(43 \%)$ de observaciones se llevaron a cabo en la hora crítica por temperatura alta, que es entre las 13:00 y 17:00 horas. La participación fue voluntaria y la distribución por edad y sexo dependió de la permanencia en la vivienda y disponibilidad ocupacional para la hora y fecha de la evaluación.

El $81 \%$ de la muestra se encontró en un rango entre 19 y 39 años de edad. El 58\% de los encuestados correspondió al sexo femenino debido a que, la mayoría de los hombres, se encontraban en el trabajo o en la escuela.

En el caso de la vivienda autoproducida las colonias estudiadas fueron: Melchor Ocampo II, Xoclán Santos y Nueva San José Tecoh. La participación fue voluntaria y la distribución por edad y sexo dependió de la permanencia en la vivienda y disponibilidad ocupacional para la hora y fecha de la evaluación; el 37\% de la muestra estuvieron en dos rangos de edad: de 13 a 19; y de 30 a 39 años, lo que dio como resultado que el $74 \%$ de las personas que respondieron fueran jóvenes. $\mathrm{El} \quad 70 \%$ de los encuestados correspondieron al sexo femenino debido a que los hombres no estaban en casa.

Se hizo un análisis de correlación de datos entre la sensación térmica percibida y la temperatura de bulbo seco, se aplicó el método MIST (Figura 4), propuesto por (GÓMEZ et al., 2007) el cual fue desarrollado con base en la propuesta de Nicol (1993) para los climas "asimétricos"; que consiste en utilizar la estadística descriptiva en la determinación de un valor neutral de temperatura u otra variable ambiental, que se considera la de confort térmico.

Los resultados aquí presentados, son parte de una primera etapa de análisis de información, y solo se aplicó el procedimiento del MIST a la temperatura de bulbo seco y no a la temperatura de globo negro, humedad relativa o velocidad de viento.

La diferencia fundamental del MIST con el método convencional es que antes de obtener la línea de regresión que caracteriza a la muestra estudiada, se determinan grupos o estratos de la misma para calcular el valor promedio y la desviación estándar de cada uno de ellos. Así, la regresión no se hace con todos los pares de datos de la muestra, sino sólo con los valores medios y los rangos se establecen mediante la adición y sustracción de una o dos veces la desviación estándar (DS) de la muestra.

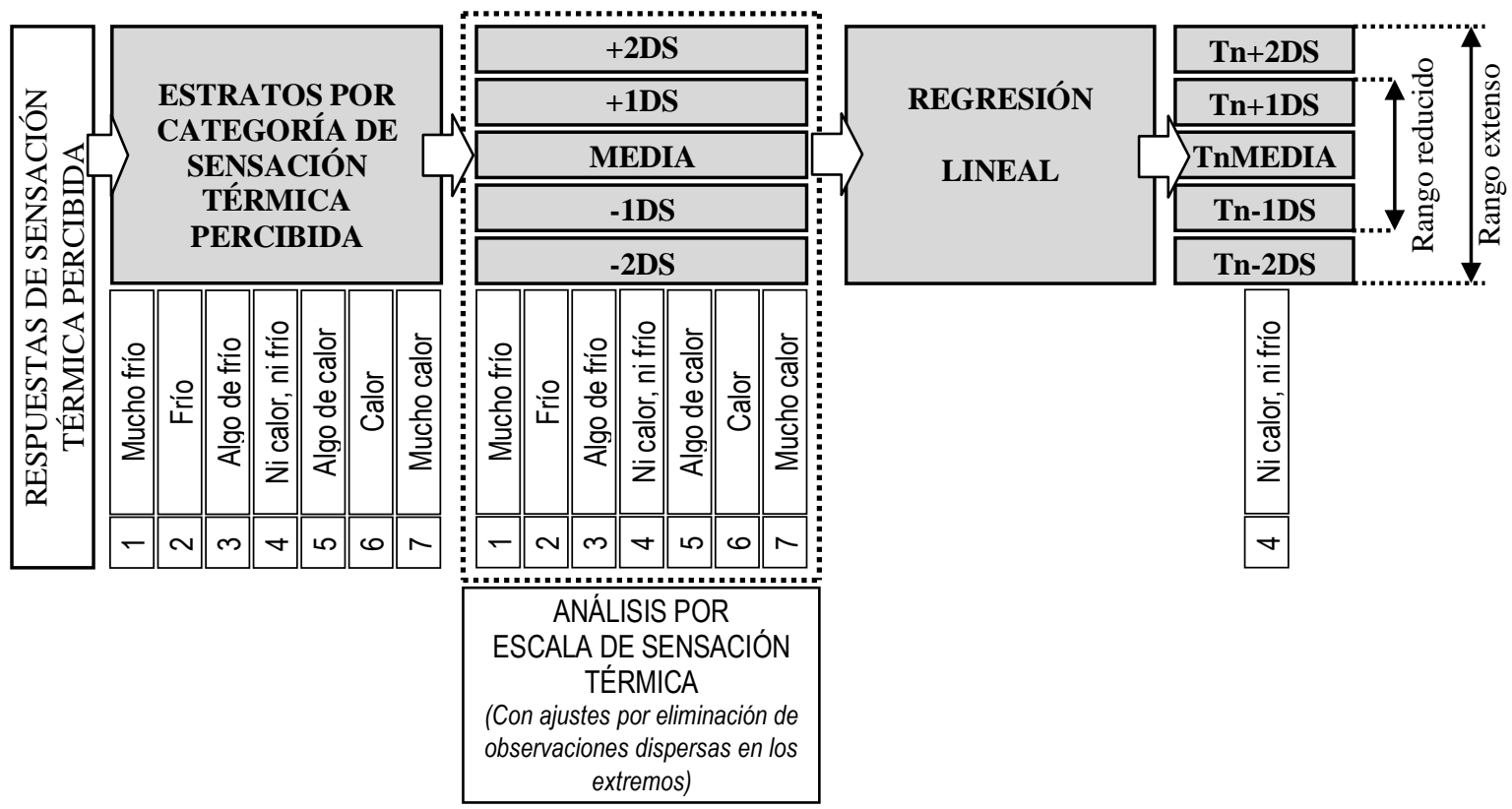

Figura 4 - Estimación de temperatura neutra (Tn) y rangos de confort térmico con el método de Medias por Intervalo de Sensación Térmica (MIST)

Fuente: Bojórquez (2010). 
La desviación estándar se emplea como una medida de la dispersión de las respuestas, y por lo tanto sirve para determinar los estratos en que se pueden ordenar éstas. Se estima que para datos normalmente distribuidos, el rango de $\pm 1 \mathrm{DS}$ incluye al $68 \%$ de las respuestas y $\pm 2 \mathrm{DS}$ incluye al $95 \%$. Para datos no distribuidos normalmente este porcentaje puede variar, por lo que se recomienda obtener el mayor número de respuestas en los estudios de campo para lograr una distribución lo más cercana a la "normal".

Para este estudio, se procesaron por separado los datos colectados en el trabajo de campo de acuerdo a cada una de las siete categorías de respuesta de confort según ISO 10551 (ISO, 1995) que son las presentadas en la Figura 4. Se determinaron para cada una de ellas los valores promedio y desviación estándar de las temperaturas registradas para cada respuesta colectada.

Posteriormente se realizó una regresión lineal con los valores que fueron obtenidos, a fin de determinar las rectas correspondientes a los límites extremos de los rangos definidos por TnMedia $\pm 2 \mathrm{DS}$, y a los límites cercanos definidos por TnMedia \pm 1 DS. También se hizo lo mismo con los valores de TnMedia.

La intersección de cada una de las líneas de regresión con la ordenada cuatro (que representa la sensación térmica de confort) determinó el valor de la temperatura neutra según el método MIST, así como los valores límites de los rangos de confort térmico. Finalmente se obtuvieron los coeficientes de determinación para los valores medios, $\pm 2 \mathrm{DS}$ y $\pm 1 \mathrm{DS}$, para efectos de validez del modelo se consideró el valor de la media.
Cabe mencionar que en este caso, el análisis desarrollado no hace diferenciaciones de edad o sexo en los sujetos de estudio, con la intención de estimar modelos genéricos de fácil aplicación para la toma de decisiones en el diseño arquitectónico. Además, el efecto de la adaptación psicológica en los sujetos de estudio fue considerado de forma esencial, en función de sus respuestas y en la escala de sensaciones subjetivas utilizadas, sin llegar a hacer un análisis específico de estos aspectos.

\section{Resultados}

La evaluación de la sensación térmica percibida, fue con base en la percepción del ambiente térmico conforme a ISO 10551 (ISO, 1995). Se analizaron:

(a) temperatura de confort térmico y amplitud de rangos;

(b) preferencia térmica; y

(c) aceptación personal del ambiente y tolerancia personal.

\section{Temperatura de confort térmico y amplitud de rangos}

La temperatura interior, en la vivienda económica, tuvo un mínimo de $19{ }^{\circ} \mathrm{C}$ y máxima de $35^{\circ} \mathrm{C}$, con valores promedio de mínima de $23{ }^{\circ} \mathrm{C}$ y máxima de $33{ }^{\circ} \mathrm{C}$. Mientras que en la vivienda autoproducida la mínima fue de $30{ }^{\circ} \mathrm{C}$ y la máxima de $38{ }^{\circ} \mathrm{C}$, con valores promedio de $33{ }^{\circ} \mathrm{C}$ de mínima y $34{ }^{\circ} \mathrm{C}$ máxima. Lo antes mencionado se presenta en la Figura 5.

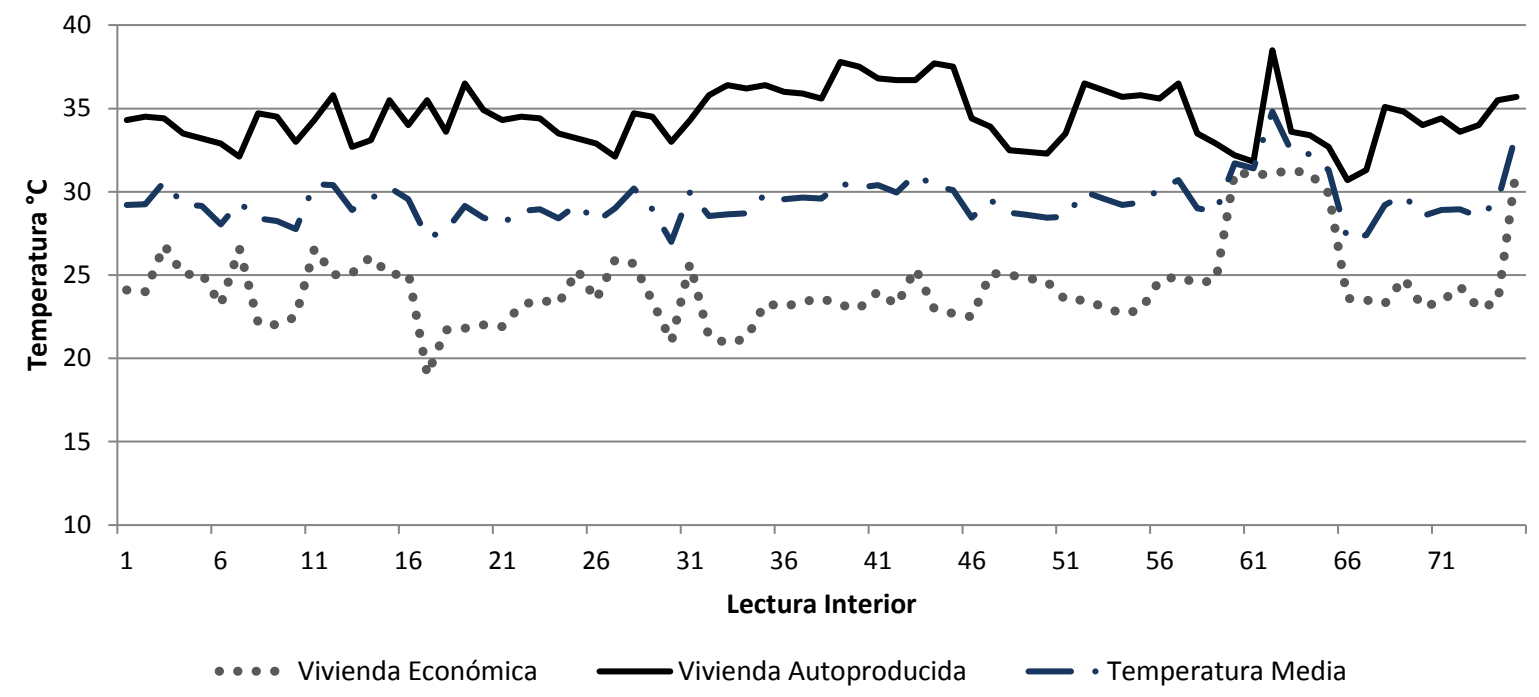

Figura 5 - Temperatura interior por tipo de vivienda 


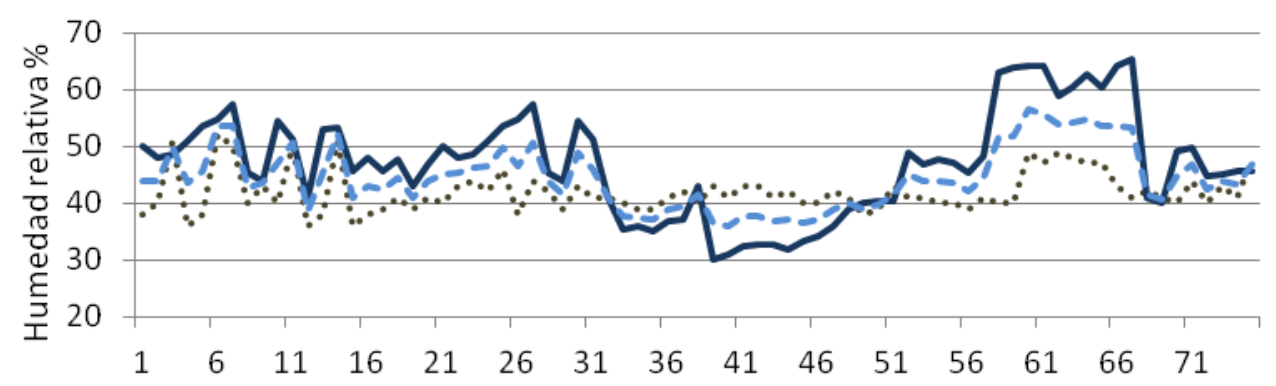

Humedad Interior

Vivienda Económica

Vivienda Autoproducida - - - - Humedad Media

Figura 6 - Humedad interior por tipo de vivienda

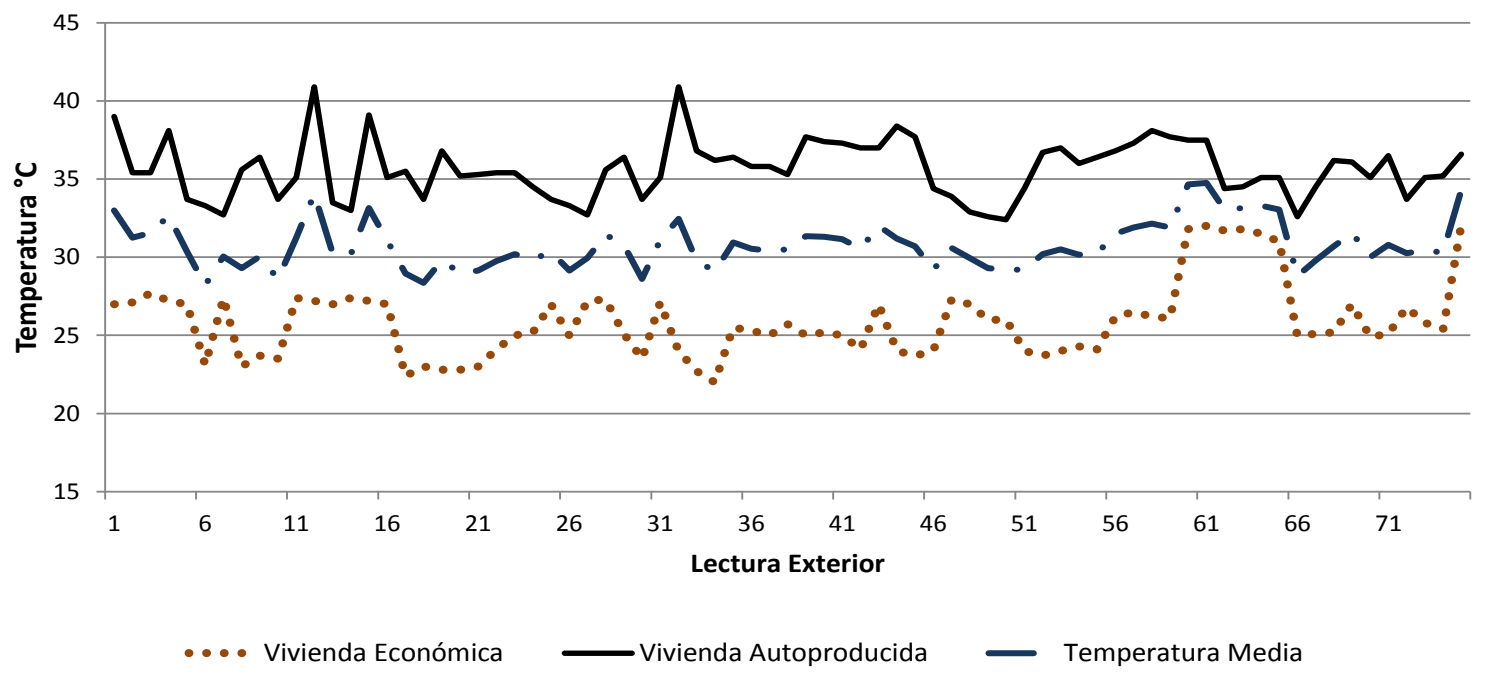

Figura 7 - Temperatura exterior por tipo de vivienda

\begin{tabular}{|l|c|c|c|}
\hline $\begin{array}{c}\text { TIPO } \\
\text { DE } \\
\text { VIVIENDA }\end{array}$ & $\begin{array}{c}\text { TEMPERATURA } \\
\text { DE CONFORT } \\
\text { TÉRMICO }\end{array}$ & $\begin{array}{c}\text { LÍMITE MÍNIMO } \\
\text { DE CONFORT } \\
\text { TÉRMICO }\end{array}$ & $\begin{array}{c}\text { LÍMITE MÁXIMO } \\
\text { DE CONFORT } \\
\text { TÉRMICO }\end{array}$ \\
\hline $\begin{array}{l}\text { Vivienda } \\
\text { económica }\end{array}$ & $32,3^{\circ} \mathrm{C}$ & $27,9^{\circ} \mathrm{C}$ & $35,1^{\circ} \mathrm{C}$ \\
\hline $\begin{array}{l}\text { Vivienda } \\
\text { auto-producida }\end{array}$ & $34,5^{\circ} \mathrm{C}$ & $28,3^{\circ} \mathrm{C}$ & $36,1^{\circ} \mathrm{C}$ \\
\hline
\end{tabular}

Quadro 1 - Temperatura y rangos de confort térmico

La humedad relativa, en la vivienda económica fue de $24 \%$ a $52 \%$ y en la auto-producida de $30 \%$ hasta $65 \%$ con lo que la sensación térmica de calor aumentó (ver Figura 6).

La temperatura exterior de las viviendas está en proporciones similares, de la económica en el rango de $22{ }^{\circ} \mathrm{C}$ a $43{ }^{\circ} \mathrm{C}$ y de auto-producida entre $32{ }^{\circ} \mathrm{C}$ a $40{ }^{\circ} \mathrm{C}$ (ver Figura 7). Cabe mencionar que las condiciones de contexto inmediato exterior en la vivienda económica son: pisos y banquetas de concreto, calles de asfalto y poca vegetación y separación de 0.90 m entre viviendas; en el caso de la vivienda autoproducida hay pisos de tierra natural, vegetación, algunas partes de concreto en banquetas con calles de tierra y la separación entre las casas es de 1.5 a $2 \mathrm{~m}$.

La temperatura de confort térmico (Tn) (ver Quadro 1), presentó una variación de aproximadamente el $7 \%\left(6{ }^{\circ} \mathrm{C}\right)$ entre ambos tipos de vivienda, los habitantes de la vivienda económica manifestaron sentirse cómodos en temperaturas hasta $32,3{ }^{\circ} \mathrm{C}$ y las personas que

106 Garcia Gomez, C.; Bojórquez Morales, G.; Ruiz Torres, P. 
viven en las viviendas auto-producidas, dijeron sentirse bien en $34,5^{\circ} \mathrm{C}$, esto representa una mayor aclimatación de estos últimos.

En lo que respecta a los rangos de confort térmico, se analizó la variación para $\pm 1 \mathrm{DS}$, ya que se considera serían los mejor adaptados por presentar respuestas más similares y con menor amplitud de rango. El límite mínimo de confort térmico presentó una variación del $1.4 \%$ entre los habitantes de vivienda económica y vivienda autoproducida. Mientras que en el caso del límite máximo la variación fue del 2,7\%. La amplitud del rango de confort térmico confirma el mayor nivel de aclimatación en los sujetos de vivienda autoproducida.

\section{Preferencia térmica}

La preferencia térmica fue evaluada en términos del porcentaje de respuestas sobre la percepción del espacio según ISO 10551 (ISO, 1995). La sensación térmica por temperatura, en la vivienda económica, presentó como predominante la sensación de "mucho calor" con un $41 \%$, mientras que en la vivienda auto-producida la sensación de porcentaje mayor fue "calor" con $46 \%$, lo que comparado con la vivienda económica, representa un valor menor en la escala de ISO 10551 (ISO, 1995). Esto confirma el mayor nivel de aceptación térmica, por parte de la población de la vivienda auto-producida, respecto a las condiciones de ambiente térmico (ver Quadro 2).

En el caso de la sensación percibida por humedad, en ambos tipos de vivienda la mayoría de las personas sintieron la sensación en la piel como "algo húmedo", sin embargo, hay cuatro puntos porcentuales más en el caso de la vivienda autoproducida, lo que indica una afectación directa de esa variable ambiental (ver Quadro 2).

La preferencia de temperatura muestra una escala menor de requerimiento para confort térmico en la vivienda auto-producida, es decir hay una mayor preferencia del ambiente térmico existente (ver Quadro 2).

Algo importante, respecto a los resultados anteriores, es que las diferencias en el ingreso económico a nivel familiar se reflejan en la utilización de ventiladores; en la vivienda económica algunas familias los encienden durante el día y noche, mientras que en la vivienda autoproducida no se utilizan.

Esto último se debe a que en la época de verano en Yucatán la energía eléctrica de uso doméstico es subsidiada por el Estado, con un límite máximo de $250 \mathrm{kWh}$ de consumo mensual (CONSEJO..., 2010). Por esta razón, en la vivienda autoproducida se restringe el gasto eléctrico y las familias procuran mantener un bajo consumo. Este fenómeno de tipo socioeconómico, repercute en la experiencia y expectativa térmica de los sujetos de estudio.

En la vivienda auto-producida, sin dispositivos de control climático, la temperatura de bulbo seco interior (Tbs) fluctúo entre 30,7 y $40,9{ }^{\circ} \mathrm{C}$ (ver Figura 8), el $44 \%$ de los casos estuvieron en el rango de 32 a $36{ }^{\circ} \mathrm{C}$ y el $56 \%$ tuvo temperatura mayor a $36,2{ }^{\circ} \mathrm{C}$. La humedad relativa (HR) fue del $30 \%$ al $65 \%$.

El $90 \%$ de la población entrevistada tenía vestimenta muy ligera, el $45 \%$ tuvo una actividad pasiva al responder la encuesta; el 51\% tenía más de media hora dentro de la casa. En lo que respecta a las condiciones de confort térmico, del total de la muestra de la vivienda auto-producida, el $39 \%$ estuvo dentro de la zona de confort térmico estimado y el $61 \%$ estuvo fuera de la misma por arriba del límite superior.

En las viviendas de tipo económico, donde los habitantes tienen un ingreso fijo, el $30 \%$ de los espacios tuvieron dispositivos de control climático activados durante la encuesta; todos fueron ventiladores de los cuales el $11 \%$ eran de pedestal y el $19 \%$ de techo.

Quadro 2 - Percepción del ambiente térmico por tipo de vivienda

\begin{tabular}{|l|c|c|}
\hline PERCEPCIÓN & $\begin{array}{c}\text { VIVIENDA } \\
\text { ECONÓMICA }\end{array}$ & $\begin{array}{c}\text { VIVIENDA } \\
\text { AUTO- } \\
\text { PRODUCIDA }\end{array}$ \\
\hline $\begin{array}{l}\text { Sensación } \\
\text { térmica }\end{array}$ & $\begin{array}{c}\text { Mucho calor } \\
41 \%\end{array}$ & $\begin{array}{c}\text { Calor } \\
46 \%\end{array}$ \\
\hline $\begin{array}{l}\text { Sensación de } \\
\text { humedad }\end{array}$ & Algo húmedo & Algo húmedo \\
\hline $\begin{array}{l}\text { Preferencias de } \\
\text { temperatura }\end{array}$ & $31 \%$ & $35 \%$ \\
\hline
\end{tabular}




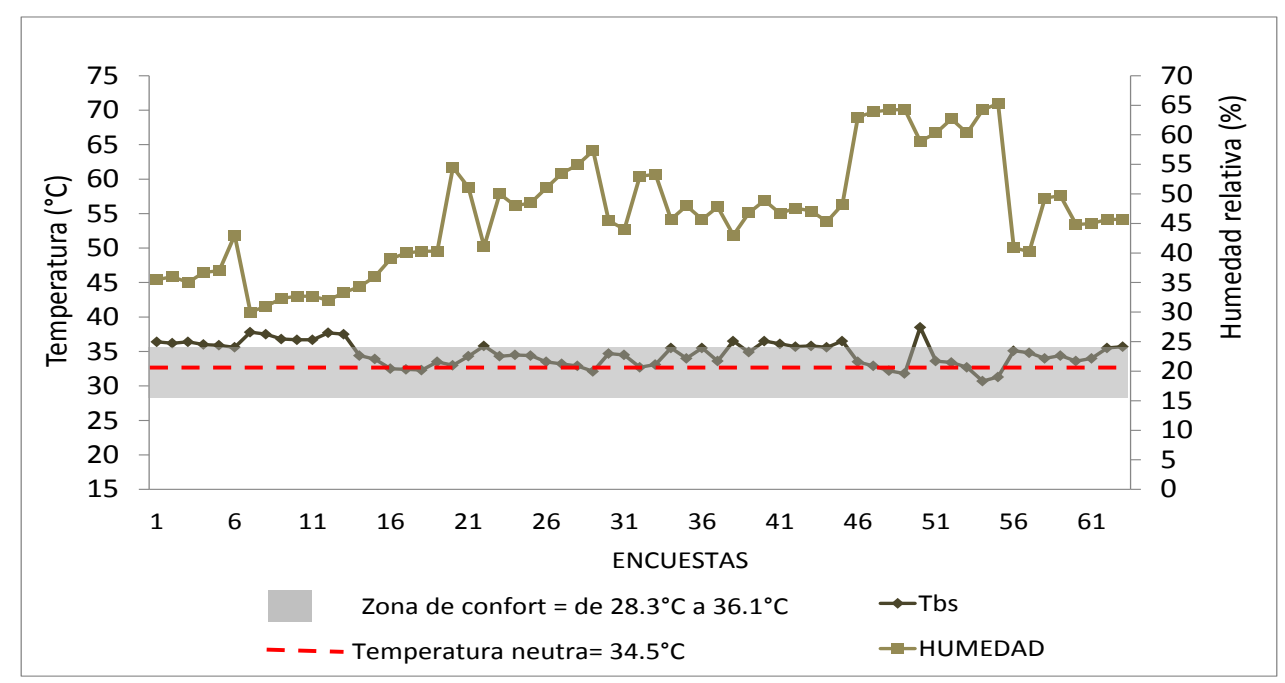

Figura 8 - Registro de temperaturas en vivienda auto-producida

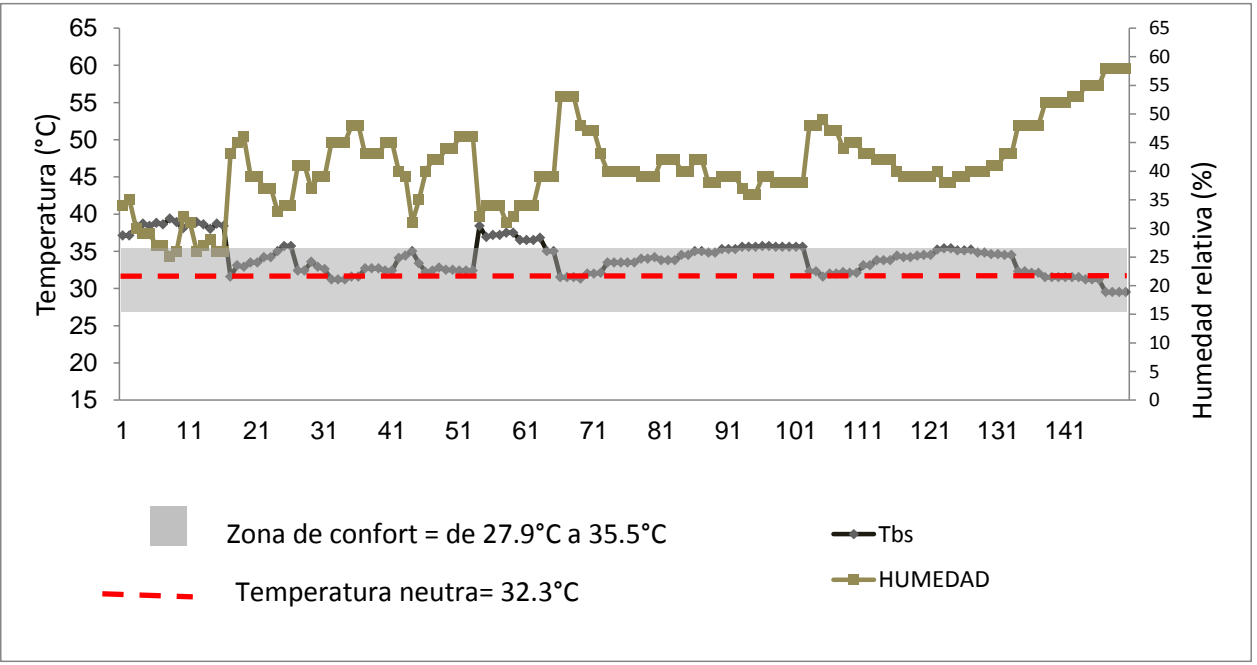

Figura 9 - Registro de temperatura en vivienda económica

La temperatura de bulbo seco interior (Tbs) estuvo en un rango de 29,5 a $39,4{ }^{\circ} \mathrm{C}$ (ver Figura 9), el $66 \%$ de las casas tuvieron lecturas en el rango de 30,0 a $35,0{ }^{\circ} \mathrm{C}$, mientras que el $31 \%$ tuvo temperatura mayor a $35,1{ }^{\circ} \mathrm{C}$. La humedad relativa (HR) fue del $25 \%$ al $58 \%$.

El $52 \%$ de la población entrevistada tenía vestimenta de tipo muy ligera; el $47 \%$ realizaban actividades pasivas al responder la encuesta y el $89 \%$ tenía más de media hora dentro de la casa.

El confort térmico dentro del edificio, en la vivienda económica el $44 \%$ estuvo dentro de la zona de confort térmico estimado y el $56 \%$ estuvo fuera de la misma por arriba del límite superior.

En términos generales se observa que la sensación térmica en la vivienda auto-producida es sólo de "calor" aunque la temperatura interior es más alta que en la vivienda económica donde las personas sintieron "mucho calor".

En los dos tipos de vivienda hubo ventilación ligera, en la vivienda económica las velocidades de viento fueron del rango de 0,2 a $0,9 \mathrm{~m} / \mathrm{s}$ en un $69 \%$, mientras que en la vivienda auto-producida la totalidad de la muestra tuvo un rango de velocidad de viento de 0,1 a $0,4 \mathrm{~m} / \mathrm{s}$. Debido a las velocidades de viento mencionadas, todas las personas prefirieron "más ventilación".

La sensación nocturna en la vivienda económica fue de "algo de calor" porque solo cuentan con dispositivos de control climático a base de ventiladores, en cambio en la vivienda autoproducida el resultado fue de "mucho calor" porque no cuentan con dispositivos mecánicos y únicamente se recurre a la ventilación natural. 
Las preferencias de temperatura son distintas en los dos tipos de vivienda; en la económica las personas quisieron sentirse "mucho más frescas" aunque la temperatura de la casa fue menor a la de las viviendas auto-producidas, donde las personas únicamente prefirieron que la vivienda fuera "más fresca".

\section{Aceptación personal del ambiente y tolerancia}

La aceptación personal del ambiente es una técnica de valoración del ambiente térmico donde se evalúa la aceptación o no de las condiciones donde se ubica el sujeto de estudio (ISO, 1995). La tolerancia personal hace referencia a los niveles de aclimatación que los habitantes tienen en los espacios de la edificación, esto se comprueba con los niveles de arropamiento que acostumbran usar los habitantes, que en este caso es distinto en los dos tipos de vivienda.

La aceptación personal del ambiente fue "generalmente aceptable" para el $62 \%$ de los habitantes de la vivienda auto-producida; mientras que para el $63 \%$ de las personas que viven en la vivienda de tipo económica fue "generalmente inaceptable" (ver Quadro 3).

Aún con las diferencias en las temperaturas, las personas de la vivienda auto-producida consideran que el ambiente térmico de su vivienda es moderadamente agradable, sin embargo en la vivienda económica la mayoría de las personas consideraron que el ambiente térmico en su vivienda es desagradable.

Esto demuestra que los habitantes de la vivienda económica están parcialmente adaptados, mientras que los de la vivienda auto-producida han aceptado condiciones de ambiente térmico con mayores temperaturas y con solo el efecto de ventilación natural nocturna como medio de conducta interactiva con el entorno.
La tolerancia personal al clima para la vivienda económica fue "ligeramente intolerable", mientras que en la vivienda auto-producida fue "tolerable" (ver Quadro 4). Lo anterior fue debido a que en espacios con calor húmedo, la evaporación se reduce, por lo que los habitantes son incapaces de disipar su calor excedente, producto del metabolismo, incrementado por el calor radiante del ambiente térmico (MONDELO et al., 2001).

\section{Conclusiones}

Se presentan condiciones de ambiente térmico diferentes en los dos tipos de vivienda, con temperatura y humedad relativa mayores en la vivienda auto-producida. Lo anterior indica un ambiente térmico con menos posibilidades de adaptación térmica en este tipo de vivienda.

Aun cuando la temperatura neutral y los rangos de confort térmico presentan valores mayores para el caso de la vivienda auto-producida en comparación con la económica, es importante mencionar que al analizar la amplitud de rangos se presenta una variación poco significativa $\left(0.06{ }^{\circ} \mathrm{C}\right)$, por lo que podría considerarse que el nivel de adaptación térmica es similar solo que bajo condiciones de ambientes térmicos diferentes.

En lo que respecta a la percepción, la sensación por temperatura, sensación por humedad y preferencia de temperatura, presentan una percepción ambiental menos crítica en el caso de la vivienda auto-producida, en comparación con los habitantes de la vivienda económica, lo que refleja una mayor aceptación de las condiciones de ambiente térmico. Sin embargo, las condiciones ambientales nocturnas tienen menor aceptación en la vivienda auto-producida, ya que en ese caso solo se cuenta con la posibilidad de ventilación natural nocturna, lo anterior indica que las condiciones nocturnas son el límite de confort térmico en la vivienda auto-producida.

\begin{tabular}{|l|c|c|}
\hline PERCEPCIÓN & $\begin{array}{c}\text { VIVIENDA } \\
\text { ECONÓMICA }\end{array}$ & $\begin{array}{c}\text { VIVIENDA } \\
\text { AUTO-PRODUCIDA }\end{array}$ \\
\hline $\begin{array}{l}\text { Aceptación } \\
\text { personal del } \\
\text { ambiente }\end{array}$ & $\begin{array}{c}\text { Generalmente } \\
\text { inaceptable } \\
63 \%\end{array}$ & $\begin{array}{c}\text { Generalmente aceptable } \\
62 \%\end{array}$ \\
\hline
\end{tabular}

Quadro 3 - Aceptación personal al ambiente térmico por tipo de vivienda

\begin{tabular}{|l|c|c|}
\hline \multirow{2}{*}{ PERCEPCIÓN } & VIVIENDA & VIVIENDA \\
& ECONÓMICA & AUTO-PRODUCIDA \\
\hline $\begin{array}{l}\text { Tolerancia } \\
\text { personal }\end{array}$ & $\begin{array}{c}\text { Ligeramente } \\
\text { intolerable } \\
51 \%\end{array}$ & $\begin{array}{c}\text { Tolerable } \\
38 \%\end{array}$ \\
\hline
\end{tabular}

Quadro 4 - Tolerancia personal al ambiente térmico por tipo de vivienda 
La evaluación del ambiente térmico, muestra una aceptación y tolerancia mayor por parte de los habitantes de la vivienda auto-producida, esto indica que se tiene no solo la aclimatación al ambiente sino también la aceptación del mismo.

Se observó una mejor adaptación térmica de los usuarios de vivienda auto-producida en comparación con las personas que habitan la vivienda económica. Lo anterior se debe a la experiencia y expectativa de ambiente térmico en la vivienda auto-producida, donde los sujetos saben que difícilmente tendrán acceso a medios electromecánicos de ventilación o enfriamiento, debido a su situación económica.

Se demuestra la capacidad de adaptación de las personas a las condiciones climáticas de Mérida, Yucatán, México y el grado de aceptación de las condiciones térmicas de los espacios de sus viviendas, que es más alto a lo establecido como límites en los estándares internacionales.

Se observó también que las preferencias térmicas de las personas difieren de acuerdo a las condiciones climáticas del lugar y sobre todo de las condiciones interiores en las que se encuentran los habitantes.

La preferencia de temperatura fue distinta en los tipos de vivienda. Esto se debe a que la adaptación ha hecho que los habitantes de la vivienda autoproducida hayan asimilado los factores del contexto y el historial térmico ha modificado sus expectativas y las preferencias del ambiente interior, con lo que se comprueba la hipótesis planteada.

\section{Referencias}

\author{
AMERICAN SOCIETY OF HEATING, \\ REFRIGERATING AND AIR CONDITIONING \\ ENGINEERS. ANSI/ASHRAE 55: thermal \\ environmental conditions for human occupancy. \\ Atlanta, 2004.
}

AYUNTAMIENTO DE MÉRIDA. Informe de Gobierno, Arq. Angélica Araujo Lara, em j de Julio de 2011.

\section{BOJÓRQUEZ, G. Confort Térmico en}

Exteriores: actividades en espacios recreativos, en clima cálido seco extremo. Tesis (Doctorado) Universidad de Colima, Colima, México, 2010.

CAO, B. et al. Field Study of Human Thermal Comfort and Thermal Adaptability During the Summer and Winter in Beijing. Energy and Buildings, v. 43, n. 5, p. 1051-1056, 2010.
CONSEJO NACIONAL DE EDUCACIÓN PARA LA VIDA EN EL TRABAJO. Cómo Calcular el Consumo de Energía Eléctrica en el Hogar.

Secretaría de Educación Pública, México.

Disponible em:

<http://www.conevyt.org.mx/recursos_multimedia /webquest/manos_ala/ahorro_energia.pdf $>$. Acceso en: 15 feb. 2010.

FANGER, O. Thermal Environment-Human Requirements. The environmentalist, v. 6, n. 4, p. 275-278, springer, 1986.

GARCÍA, E. Modificaciones al Sistema de Clasificación Climática de Köppen: para adaptarlo a las condiciones de la República Mexicana. México, D.F.: Edit. Universidad Nacional Autónoma de México, 1981.

GARCÍA-GÓMEZ, C. Un Diagnóstico de los Niveles de Consolidación de Vivienda en la Periferia de Mérida. Cuadernos Arquitectura de Yucatán, v. 13, p. 19-29, 2000.

GARCÍA-GÓMEZ, C. El Proceso de Consolidación de la Vivienda Auto-Producida en la Zona Urbana de Mérida, Yucatán. Crisol, v. 2, n. 1, p. 31-73, 2006.

GARCÍA-GÓMEZ, C. La Toma de Decisiones y el Confort térmico de la Vivienda AutoProducida en Mérida, Yucatán. Tesis (Doctorado) - Universidad de Colima, Colima, México, 2009.

GARCÍA-PERALTA, B. Vivienda Social en México (1940-1999): actores públicos, económicos y sociales. Cuadernos de Vivienda y Urbanismo, v. 3, n. 5, p. 34-49, 2010.

GÓMEZ, G. et al. Monitoreo de Condiciones de Confort Térmico. Reporte Técnico CONAFOVI. 2004-01-20. Colima, 2007.

KWOK, A; G.; RAJKOVICH, N. B. Addressing climate change in comfort standards. Building and Environment, v. 45, n. 1, p. 18-22, 2010.

INTERNATIONAL ORGANIZATION FOR STANDARDIZATION. ISO 9920: ergonomics of the thermal environment: estimation of thermal insulation and water vapour resistance of a clothing ensemble. Ginebra, 2007.

\section{INTERNATIONAL ORGANIZATION FOR} STANDARDIZATION. ISO 7730: ergonomics of the thermal environment: analytical determination and interpretation of thermal comfort using calculation of the PMV and PPD indices and local thermal comfort criteria. Ginebra, 2005. 
INTERNATIONAL ORGANIZATION FOR

STANDARDIZATION. ISO 8996: ergonomics of thermal environment: determination of metabolic heat production. Ginebra, 1995.

INTERNATIONAL ORGANIZATION FOR STANDARDIZATION. ISO 10551: ergonomics of thermal environment: assessment of the influence of the thermal environment using subjective judgment scales. Ginebra, 1995.

MONDELO, P. et al. Ergonomía 2: confort y estrés térmico. 3. ed. Barcelona: Universitat Politécnica Catalunya, 2001.

NICOL, F. Thermal Comfort "A Handbook for Field Studies Toward an Adaptive Model". London: University of East London, 1993.

PUEBLA, C. Del Intervencionismo Estatal a las Estrategias Facilitadoras: los cambios de la política de vivienda en México, 1972-1994.

México: El Colegio de México, 2002.

SINGH, M. K.; MAHAPATRA, S.; ATREYA, S. K. Adaptive Thermal Comfort Model for Different Climatic Zones of North-East India. Applied Energy, v. 88, n. 7, p. 2420-2428, jul. 2011.

\section{Agradecimientos}

Al M. Arq. Omar Ruiz Salazar, M. Arq. Andrés Quiroa, Dr. Gabriel Castañeda Nolasco, Dra. Glacir Teresinha Frike, Dr. Silvino García Galeana y colaboradores en el trabajo de campo y procesamiento de datos por el apoyo brindado para la realización de este documento. Al proyecto "Confort térmico y ahorro de energía en la vivienda económica en México: regiones de clima cálido seco y húmedo", Clave CONAFOVI 200401-20; al proyecto "Desarrollo de una vivienda ecológica autosustentable", Clave YUC-2008-C06107327; al Programa Interinstitucional de Doctorado en Arquitectura, a la Universidad Autónoma de Yucatán, Facultad de Arquitectura; a la Universidad Autónoma de Baja California, Facultad de Arquitectura y Diseño y a la Universidad Autónoma de Chiapas, Facultad de Arquitectura.

Revista Ambiente Construído

Associação Nacional de Tecnologia do Ambiente Construído

Av. Osvaldo Aranha, $99-3^{\circ}$ andar, Centro

Porto Alegre - RS - Brasil CEP $90035-190$

Telefone: +55 (51) 3308-4084

Fax: +55 (51) 3308-4054

www.seer.ufrgs.br/ambienteconstruido

E-mail: ambienteconstruido@ufrgs.br 